\title{
Investigation and identification of foreign turbidity particles in beverages via Raman micro-spectroscopy
}

\author{
Eva-Maria Kahle $^{1}$ (D) Martin Zarnkow ${ }^{1} \cdot$ Fritz Jacob $^{1}$
}

Received: 1 September 2020 / Revised: 24 October 2020 / Accepted: 31 October 2020

(c) The Author(s) 2020

\begin{abstract}
Cloudiness, opalescence or a milky appearance in beverages is usually undesirable and lead the consumers to assume that the product is of lower quality. Many different types of formation and entry can lead to cloudiness and these causes can be divided into two major categories: beverage-specific, where the ingredients cause an interaction, and external influences such as process errors or particles interacting with the medium. This study considers two main sources of external influences. Raman micro-spectroscopy (RMS) was used to detect, evaluate and validate filter aids, stabilisers and various microplastic (MP) particles. A suitable sample preparation was developed, membrane filters were tested, and a filtration method for isolating the individual particles was established and implemented. To identify particles with RMS and for better representation, a few particles were selected and the results were validated using cluster analysis and the similarity matrix. The different media influences were identified by analysing the particles both dry, in water and beer. The filtration residue after membrane filtration was also analysed. A two-dimensional image scan of the particles served to determine particle homogeneity. The spectra were then recorded with single-point scans. The polyvinylpolypyrrolidone (PVPP) spectra in the different media showed similarities greater than $80 \%$, usually greater than $95 \%$. The cellulose spectra showed no differences between the different media, but consistently high average similarities of $94.5 \%$. This investigation should show that foreign particles can be detected and evaluated by RMS with suitable sample preparation and recording.
\end{abstract}

Keywords Turbidity $\cdot$ Beer $\cdot$ Beverages $\cdot$ Filter aids and stabilisers $\cdot$ Microplastic $\cdot$ Raman

\section{Introduction}

In addition to the turbidity that occurs due to the raw material, the colloids that cause turbidity must also be taken into account, which are caused by particles that are foreign to beer. The presence of beer-extraneous turbidity particles usually indicates errors in process control, e.g. when particles from filter aids or stabilising agents break through the filter medium due to pressure surges. In addition to filter aids and stabilisers, other particles with the potential to form turbidity can also occur in breweries [1-4]. In this context, label fibres, lubricant residues from the lids of beer cans as well as plastic abrasion or microplastics from conveyor belts, membranes, valves, pipes or seals should be mentioned. The

Eva-Maria Kahle

eva.maria.kahle@tum.de

1 Forschungszentrum Weihenstephan für Brau- und Lebensmittelqualität, Technische Universität München, Alte Akademie 3, 85354 Freising, Weihenstephan, Germany
MP particles can cause turbidity from the product interacting with the packaging $[4,5]$. With the development of new analytical methods and techniques, it is possible to analyse raw materials and food during operation. Especially in the case of beverage samples, there is usually no risk of MP being introduced via the raw materials due to the frequently filtration steps in the closed process. Instead, most particles in beverages can be attributed either to exposure through process plastic during production or to subsequent contamination as a result of unsuitable sampling and treatment methods [6]. The plastic can enter the beer during the process, e.g. through the manual addition of additives and raw materials, contaminated water in the brewhouse and fermentation cellar. It can also enter the beer during transport and storage by dissolving crown cork compound materials or PET from the wall of a plastic bottle [7, 8]. According to the definition of the National Oceanic and Atmospheric Administration, MP are plastic particles with a size of 0.1-5000 $\mu \mathrm{m}$ [9].

Inorganic substances can cause turbidity and may include filter aids that have penetrated or label residues. 
It is also possible for organic substances of microbiological or non-microbiological origin to impair the colloidal stability of beer [4]. Turbidity of non-microbiological origin can also be distinguished as cold turbidity, permanent turbidity and turbidity from foreign substances [10]. As is the case for non-alcoholic beverages, turbidity can occur in beer in three different ways: Particles already contained in beer or its raw materials can coagulate, and turbidity can also arise from substances introduced during beer production. Last but not least, foreign particles can enter the beer before or during filling and cause turbidity [4]. Figure 1 lists various types of turbidity and shows their origins. The overview categorises the different origins of turbidity. In this study, the beer-extraneous turbidity particles are a particular focus and will be explained in more detail in the following sections. By means of Raman micro-spectroscopy (RMS), numerous filter aids (cellulose, kieselguhr, perlite) and stabilisers (PVPP, silica gel like xerogel) as well as the most common MP particles (UP, PA, PE, PF, PMMA, PS, PVC, PP, PVDF, PTFE, PEEK, PET) were detected, evaluated and validated. At the beginning, a suitable sample was prepared with filtration equipment and a filter validation was performed to detect the foreign particles also in the liquid medium.

\section{Materials and methods}

The focus of this work is on the identification and characterisation of non-beer turbidity particles. Beer is a highly complex medium comprising more than 450 ingredients [11-14]. This complex matrix often makes the analysis of different particles difficult using Raman spectroscopy, since the substances to be analysed are often overlaid by other ingredients [15]. Therefore, it is important to establish a suitable sample preparation in advance of the analysis to solve these matrix problems. Three various filter aids and stabilisers as well as twelve plastics were analysed, which are described in detail in the following. Samples were prepared in five-fold determination $(n=5)$ for the replicates.

\section{Filter aids and stabilisers}

In terms of filter aids, kieselguhr (Dicalite Speedflow), perlite (Dicalite MF2) and cellulose fibres (CelluFluxx P 50) were used as sample materials, which were provided by Erbslöh $\mathrm{GmbH}$, Geisenheim. The kieselguhr used is a preparation of medium fineness. Dicalite MF2 is a medium to coarse perlite and CelluFluxx P 50 is an extra-long cellulose fibre. All preparations were stored in a dry and dark location at room temperature. Preparations from various manufacturers were used for the stabilisers. We analysed a non-regenerable

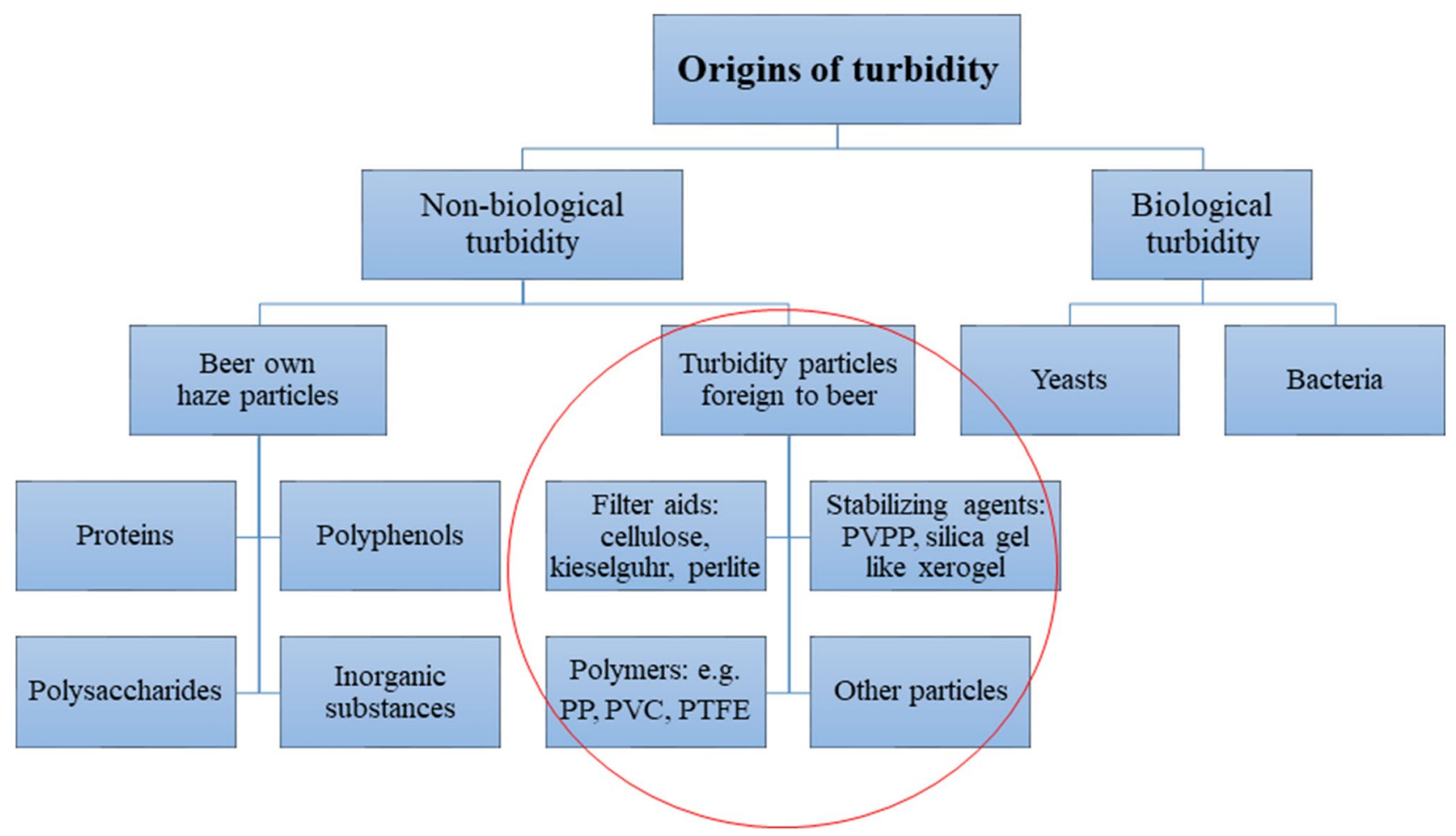

Fig. 1 Various origins of turbidity. For this investigation the turbidity particles foreign to beer are relevant 
PVPP from Erbslöh GmbH, Geisenheim and used the PVPP preparation Stabiclear from Stabifix Brauerei-Technik KG and also used the silica gel Stabifix Extra. This is a hydrated xerogel. All preparations were stored in a dry place and protected from light at room temperature.

\section{Sample preparation of filter aids and stabilisers}

To investigate the influence of different media on the Raman spectra, the PVPP preparations of both manufacturers (Stabifix and Erbslöh), as well as the cellulose preparation were analysed as a dry powder, a suspension in water, a suspension in beer and as a filtration residue after membrane filtration. To prepare the suspension in water, $2 \mathrm{mg}$ each of the dry PVPP and cellulose preparation was weighed and suspended in $1000 \mu \mathrm{L}$ sterile water using a vortex mixer. The suspension in beer was prepared in the same way using beer instead of water. The beer was first decarbonised by shaking. Furthermore, a standing time of two hours before starting measurement to allow for possible enrichment of the PVPP particles with polyphenols from the beer. To analyse the filtration residue, a suspension in beer was also prepared. A suspension of $7.5 \mathrm{mg}$ PVPP was suspended in $150 \mathrm{~mL}$ beer, which corresponds to a dose of $5 \mathrm{~g} / \mathrm{hL}$. In the case of the cellulose preparation, $37.5 \mathrm{mg}$ cellulose fibres were suspended in $150 \mathrm{~mL}$ beer, which corresponds to a dosage of $25 \mathrm{mg} / \mathrm{hL}$.

\section{Polymers (MP particles)}

To cover the widest possible range of plastic classes, a collection of plastic samples from PlasticsEurope Deutschland e.V. were used. PET samples in different trade forms were also provided by Forum PET for this study. Table 1 lists the plastic workpieces and shows an overview of all the examined plastic samples, product samples and materials, as well as the origin of the samples.

\section{Sample preparation of polymers}

To validate the analytical method, the existing plastic samples were crushed by rubbing them with different grades of sandpaper. The resulting spectra were stored so that the polymer spectra could be corrected if necessary. The consequential particles were then transferred to screw-top test tubes as storage containers and could be removed with a spatula if necessary. The abrasive paper was previously segmented to avoid cross-contamination of the polymer types. At the beginning, no uniform particle size distribution was required, so the plastic dusts could be used as internal quality standards. To avoid interfering signals, the sandpaper material was examined microscopically and spectroscopically. The resulting spectra were stored so that the polymer spectra could be corrected if necessary. The microplastic preparations produced in this way are shown in Fig. 2 in the sequence shown in Table 1, at fourfold magnification.

\section{Filtration and validation}

The membrane filters were cut into quarters for microscopic and spectroscopic validation of the filter surface and fixed between two uncoated slides. In doing so, alignment had to be observed so as not to confuse the front and back sides of the gold-coated membrane filters. To analyse the polymer samples on the filter surfaces, $20 \mathrm{~mL}$ screw-top test tubes were cleaned according to the procedure described above and stored upside down in a fume cupboard for drying until use. Subsequently, $100 \mathrm{mg}$ of each of the various particle dusts was weighed into the screw-top test tubes and filled with $10.0 \mathrm{~mL}$ of double-distilled water [9]. The tops were screwed on to the tubes, which were suspended in a vortex mixer directly before being transferred for filtration. The vacuum pump was then switched off and the membrane filter was transferred to a slide using special tweezers.
Table 1 Analysed polymer types and their origin

\begin{tabular}{llll}
\hline Sample no. & Material & Abbreviation & Origin \\
\hline 1 & Unsaturated polyester resin & UP & PlasticsEurope \\
2 & Polyamide & PA & PlasticsEurope \\
3 & Polyethylene & PE & PlasticsEurope \\
4 & Phenolic resin & PF & PlasticsEurope \\
5 & Polymethyl methacrylate & PMMA & PlasticsEurope \\
6 & Polystyrene & PS & PlasticsEurope \\
7 & Polyvinyl chloride & PVC & PlasticsEurope \\
8 & Polypropylene & PP & Reed valve housing \#1 \\
9 & Polyvinylidene fluoride & PVDF & Reed valve housing \#3 \\
10 & Polytetrafluoroethylene & PTFE & valve \#6 \\
11 & Polyetheretherketone & PEEK & Seat gasket \#8 \\
12 & Polyethyleneterephthalate & PET & Forum PET \\
\hline
\end{tabular}




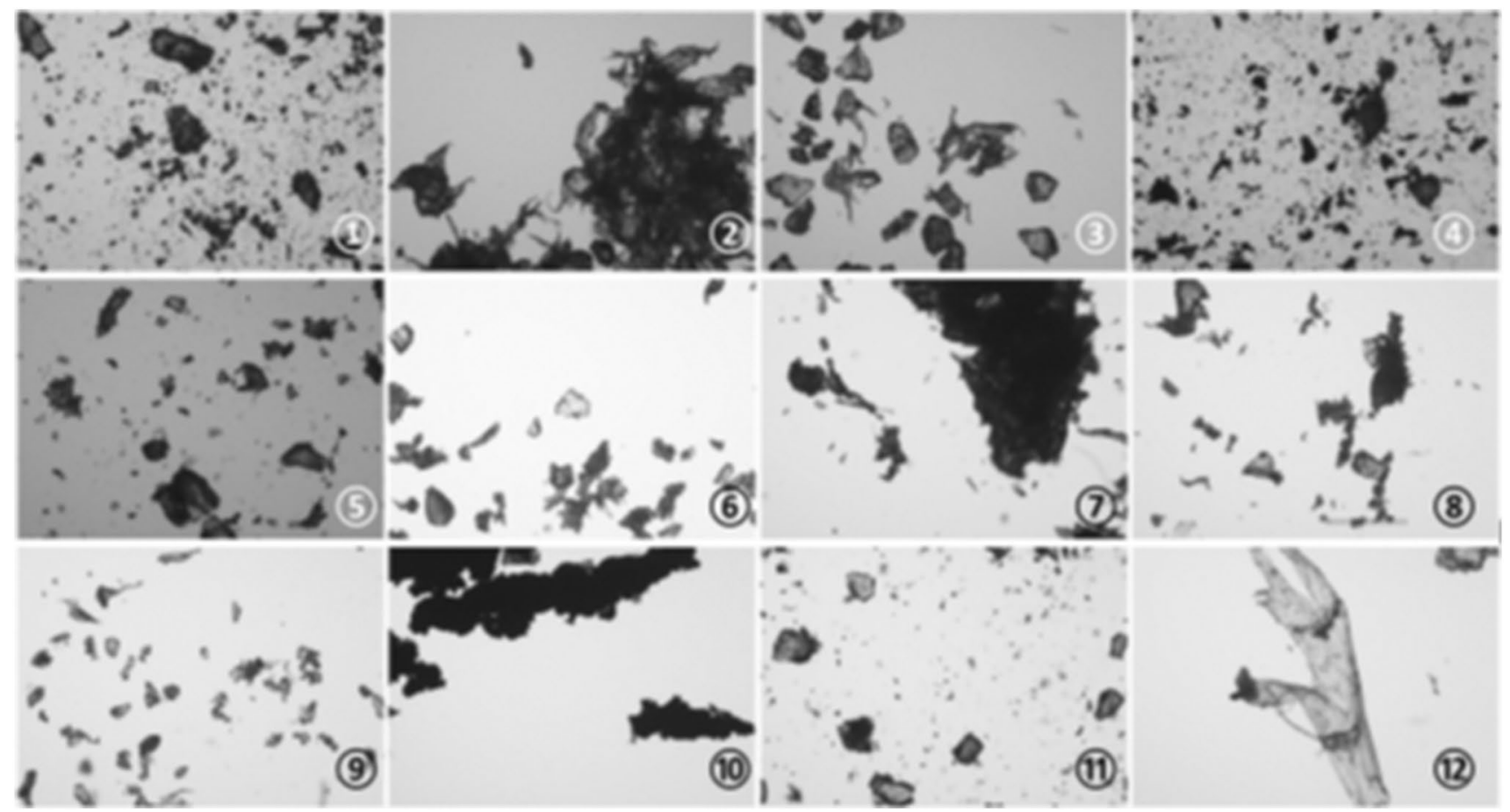

Fig. 2 Microscopic images of the plastic samples used at $4 \mathrm{x}$ magnification; labelling according to Table 1

To check the suitability of the membrane filters for routine analysis, all filters were examined microscopically and spectroscopically. First, the requirements for the filter membranes were checked for each filter type. For this purpose the filters were analysed for irregularities in their surface structure using bright-field microscopy with the $20 \times$ lens. The Raman spectra of the individual filters were then recorded. The same settings were selected as for the polymer reference spectra. The thermal stability of the filters was also qualitatively determined. To determine the maximum laser intensities and thus the thermal stability of the individual filter types and materials, Raman spectra of the filters were recorded at 2, 5, 10, 20 and $40 \mathrm{~mW}$ laser power. The thermal resistance was defined and validated.

\section{Sampling and preparation}

The principle of due diligence applies in particular to the process steps for obtaining and preparing samples, as well as all other steps preceding the analysis. The theoretical analysis limits of the common analysis methods for microplastics are currently between $20 \mu \mathrm{m}$ (FTIR), $10 \mu \mathrm{m}$ (NIR) and $1 \mu \mathrm{m}$ (RS) $[15,16]$.

\section{Evaluation of membrane filters}

Some pre-analysis preparation is necessary for smaller MP particle sizes, for example, filtering the particles and removing the sample matrix. To avoid interfering signals superimposing on the particle Raman signals, high demands are placed on the filter material and the filter parameterisation. Depending on the complexity of the sample, filter specifications and analysis parameters and smaller particle sizes can be measured.

\section{Membrane filtration}

To validate suitable filter materials and surfaces, five membrane filter types were tested from Analytische Produktions-, Steuerungs- und Controllgeräte GmbH (APC) and three membrane filter types from i3 Membrane $\mathrm{GmbH}$. These eight membrane filters had different filter materials, as well as different layer thicknesses or the arrangement of the gold coating. The membrane filters were stored in the storage containers provided and protected from light and contamination. The relevant specifications of the tested filters are listed in Table 2.

The membrane filters used, however, had a diameter of $25 \mathrm{~mm}$ due to given test parameters. The sample preparation and a suitable filtration attachment was designed and manufactured. The requirements placed on the workpiece are listed in Table 3.

The preliminary design drawing is shown in Fig. 3. For the basic construction, standardised stainless steel components of the company Martin Wagner "WAGNERINOX" were used. Subsequently, a tapered stainless steel work 
Table 2 Material, coating properties and manufacturer of the validated membrane filters

\begin{tabular}{lllll}
\hline No & Description & Filter carrier material & $\begin{array}{l}\text { Arrangement of } \\
\text { the coating }\end{array}$ & Manufacturer \\
\hline 1 & APC Thicker Type & Polycarbonate & $40 / 20$ & APC GmbH \\
2 & APC PET & Polyethyleneterephthalate & $40 / 20$ & APC GmbH \\
3 & APC Folie 1 & Filter film 1 & $40 / 20$ & APC GmbH \\
4 & APC Folie 2 & Filter film 2 & $40 / 20$ & APC GmbH \\
5 & APC PC & Polycarbonate & $0 / 0$ & APC GmbH \\
6 & I3 Trackpor Gold & Polycarbonate & $40 / 20$ & i3 Membrane GmbH \\
7 & I3 Trackpor Gold & Polycarbonate & $40 / 0$ & i3 Membrane GmbH \\
8 & I3 Flexipor & Aluminium oxide & $0 / 0$ & i3 Membrane GmbH \\
\hline
\end{tabular}

\begin{tabular}{ll}
\hline Requirement & Solution \\
\hline Fit for $\emptyset 25 \mathrm{~mm}$ membrane filter & More favourable design due to standardised component \\
Stainless steel body & No plastic parts on the retentate part $\rightarrow$ \\
& No additional source of contamination \\
Smooth, non-static surface & Electrochemical properties of plastic particles $\rightarrow$ Adhe- \\
& sion, falsification of results \\
Compatibility & Can be adapted to existing membrane filtration systems \\
Fast filtration, few steps & Work to exclude contamination as much as possible
\end{tabular}

Table 3 Requirements for membrane filtration
When using the $785 \mathrm{~nm}$ laser, a spectroscope of the model UHTS 400 NIR is used. This spectroscope is specially adapted to the wavelength range of $650-1300 \mathrm{~nm}$ and thus to the near infrared range. However, since the efficiency of the CCD detectors is directly dependent on the wavelength of the laser, and the Raman signal decreases by four times the power of the laser wavelength, the acquisition of Raman spectra with the $785 \mathrm{~nm}$ laser requires a longer measurement time than the $532 \mathrm{~nm}$ laser. Furthermore, the theoretically possible resolution of the laser increases with increasing wavelength, which contradicts a quantitative analysis [17, 18]. For this reason, the $785 \mathrm{~nm}$ laser is not used in this paper. Gratings scatter the detected Raman signal over the CCD detectors. The number of ridges per millimetre determines the resolution of the Raman signals and the absolute measuring time. The higher the number of ridges, the higher the resolution and the longer the analysis time. With the existing system it was possible to choose between two gratings (600 and $1800 \mathrm{~g} / \mathrm{mm}$ ). Due to the homogeneity of the samples and the measurement duration, only the $600 \mathrm{~g} / \mathrm{mm}$ grating was used. The scattered photons were then recorded by two CCD detectors from WiTec $\mathrm{GmbH}$. These convert the detected photons into electrical signals and feed the software with the data of the Raman signals. By cooling down to a working temperature of $-60{ }^{\circ} \mathrm{C}$, interfering signals (e.g. noise or dark current) are avoided. The standard intensity of the $532 \mathrm{~nm}$ laser was set at $2.0 \mathrm{~mW}$ and increased to $5.0 \mathrm{~mW}$ if necessary. The integration time per recorded spectrum wavelengths in the visible range (approx. $450-700 \mathrm{~nm}$ ). 


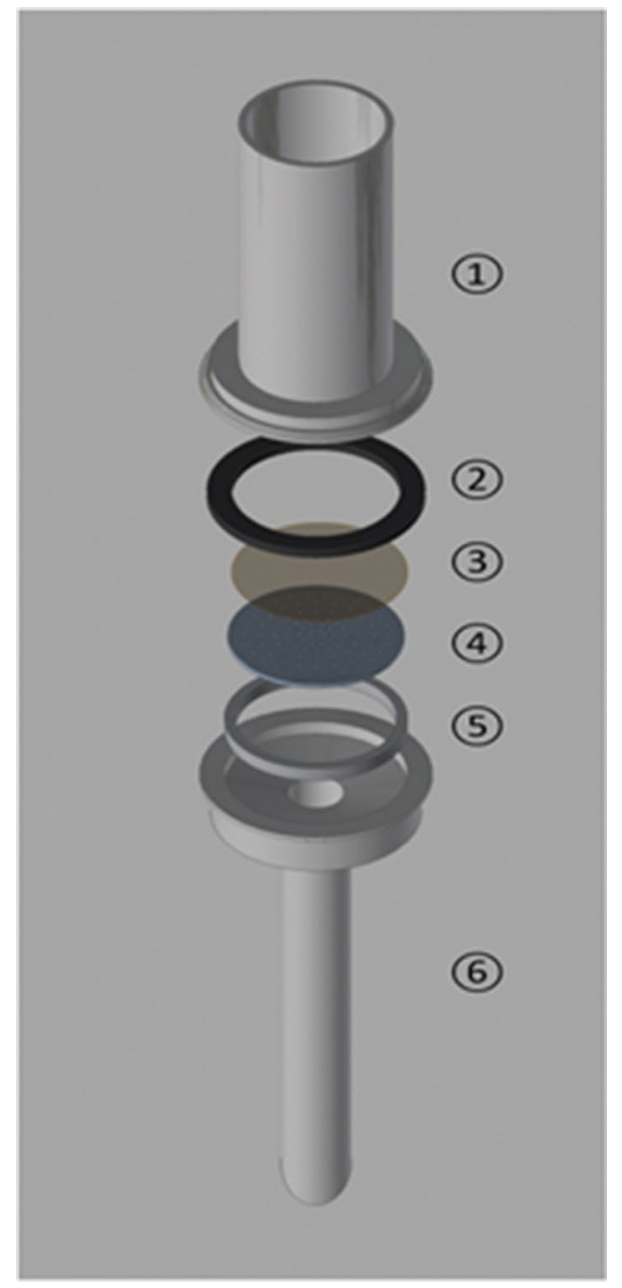

Fig. 3 Construction drawing of the filtration attachment (CAD image file); (1) The upper part is composed of a stainless steel hollow cylinder (DN25) and a clamp weld-on socket (2) The sealing ring provides the necessary contact pressure of the membrane filter (3) The membrane filter is inserted between the sealing ring and filter frit (4) The filter frit serves as a carrier surface for the membrane filter and defines the flow volume (5) The carrier ring serves as a support and fixation of the filter frit (6) The lower part is composed of a bevelled stainless steel hollow cylinder (DN10) and a clamp welding socket

was $0.4 \mathrm{~s}$ at 50 accumulations. This results in an absolute measurement duration of $20 \mathrm{~s} /$ particle. To obtain significant reference spectra, five individual spectra of each substance were recorded at different positions within a sample and an average spectrum was generated. Video images of each substance were stored to be able to make comparisons during later microscopic analyses. The images were acquired with the WiTec software Control 5.1, which directly links the video images with the acquired spectra. The programme also stores the position and parameters of the single spectra, line scans and image scans. During the later evaluation the video images can be combined with the spectral data and the results can be visualized.

\section{Data analysis and statistical evaluation}

All analyses and measurements were controlled and performed with the WiTec Control FIVE 5.1 software. The quantitative data evaluation and processing was carried out by the WiTec software Project FIVE 5.1. The data material was evaluated using the software BioNumerics 7.6.3. Applied Maths N.V., Belgium. The determined average spectra of the reference samples, all spectra as well as the cluster data sets from the image scans were imported into the database and processed. A five-fold determination $(n=5)$ of all analysed samples was carried out, each with three different particles in the sample. Due to the size of the particles, the five-fold determination of the samples was carried out at three different positions within a particle. Each individual spectrum was automatically formed from the mean value of ten repeated measurements using the WITec Control software.

\section{Results and discussion}

\section{Filter aids and stabilisers}

The first peak in the Raman spectra at a wavenumber of approx. 0 rel. $\mathrm{cm}^{-1}$ can be neglected, since this peak represents the laser [19]. For a better overview, only one of the Raman spectra from each of the five samples is shown below, unless otherwise described.

\section{Samples containing cellulose and silicon dioxide}

The cellulose fibres were analysed in dry state, as suspension in water, as suspension in beer and as filtration residue. Although cellulose fibres have no adsorption capacity, it is necessary to check the particles from the filtration residue to determine whether a change in the Raman spectra can be detected as a result of a possible deposition of beer constituents. Figure 4 shows a microscope image of the cellulose fibres at $60 \times$ magnification and the corresponding spectra.

The Raman spectra of cellulose fibres show a peak at approx. 2900 rel. $\mathrm{cm}^{-1}$. At this wave number, the C-H compounds from the hydrocarbon chains of cellulose oscillate $[17,19]$. However, a characteristic oscillation frequency in the range of 2900-3500 rel. $\mathrm{cm}^{-1}$ was found for $\mathrm{OH}$ groups, so that these are also reflected in the measured Raman spectra at approx. $2900 \mathrm{rel} . \mathrm{cm} \mathrm{cm}^{-1}$ [17]. The other peaks in the range of about $600-1500 \mathrm{rel} . \mathrm{cm}^{-1}$ can generally be assigned to aliphatic hydrocarbons (600-1300 rel. $\mathrm{cm}^{-1}$ ) and organic carbon $\left(1300-1700\right.$ rel. $\left.\mathrm{cm}^{-1}\right)[17,19]$. The spectra show similarities of at least $89.6 \%$ to each other. No other groups 
Fig. 4 Microscope image of cellulose fibres in bright field with white light source at $60 \mathrm{x}$ magnification (left); Raman spectra of cellulose fibres at different sample preparations [(dry (1); suspension in water (2); suspension in beer (3); filtration residue (4) (colour-coded)], $n=10, \lambda=532 \mathrm{~nm}$ (right)
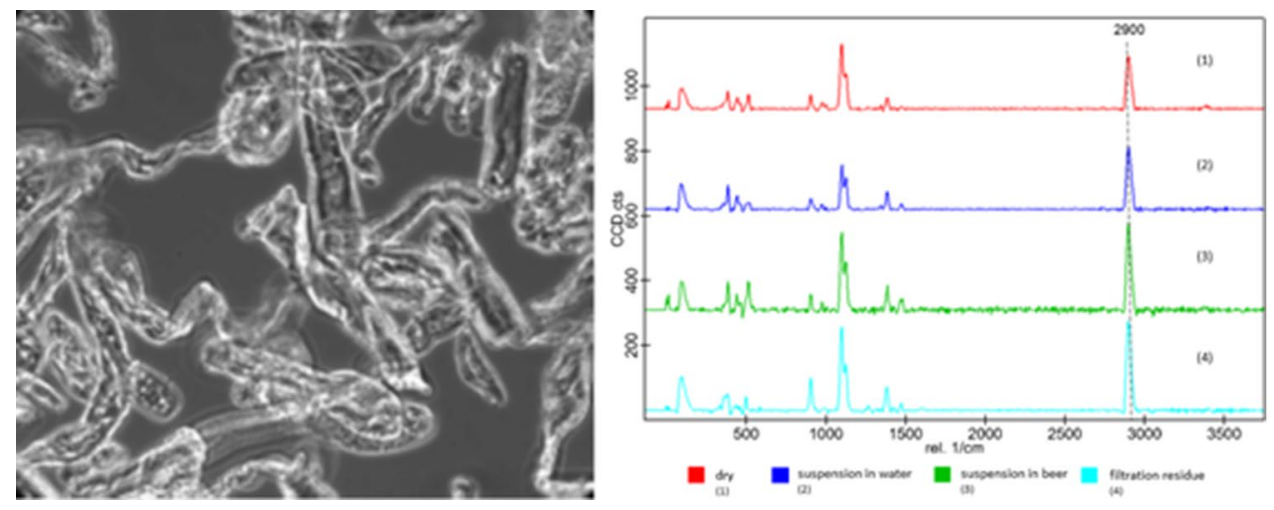

can be identified with regard to the different sample preparations, which is also confirmed by the dendrogram shown in Fig. 5.

Figure 5 shows that, due to the high similarities across all individual spectra, no clusters are formed according to the different sample preparations. The spectra shows an average of at least $94.5 \%$ similarity to each other. Thus, although cellulose fibres in different media and states cannot be distinguished from each other, all spectra show a similar Raman spectrum. Figure 6 shows microscopic images of particles of the silica gel, kieselguhr and perlite preparations investigated. The spectra each have a peak at approx. $925 \mathrm{rel} . \mathrm{cm}^{-1}$ and a slightly lower peak at approx. $460 \mathrm{rel} . \mathrm{cm}^{-1}$. In the literature, oscillation frequencies at 450-550 rel. $\mathrm{cm}^{-1}$ and $900-1100$ rel. $\mathrm{cm}^{-1}$ are assigned to the silicon oxides $[17,19]$.

Table 4 shows the $\mathrm{SiO}_{2}$ contents of hydrated xerogel, flux-calcined kieselguhr, perlite, and borosilicate glass.

It is evident that all materials have a high proportion of $\mathrm{SiO}_{2}$ in common. The spectra of the different materials are at least $91.2 \%$ similar to each other. The Table 4 also shows that the particle spectra in particular do not show
Fig. 5 Evaluation of Raman spectra of cellulose fibres at different sample preparations (colour-coded) using a hierarchical cluster analysis, $\lambda=532 \mathrm{~nm}$ similarity in \%

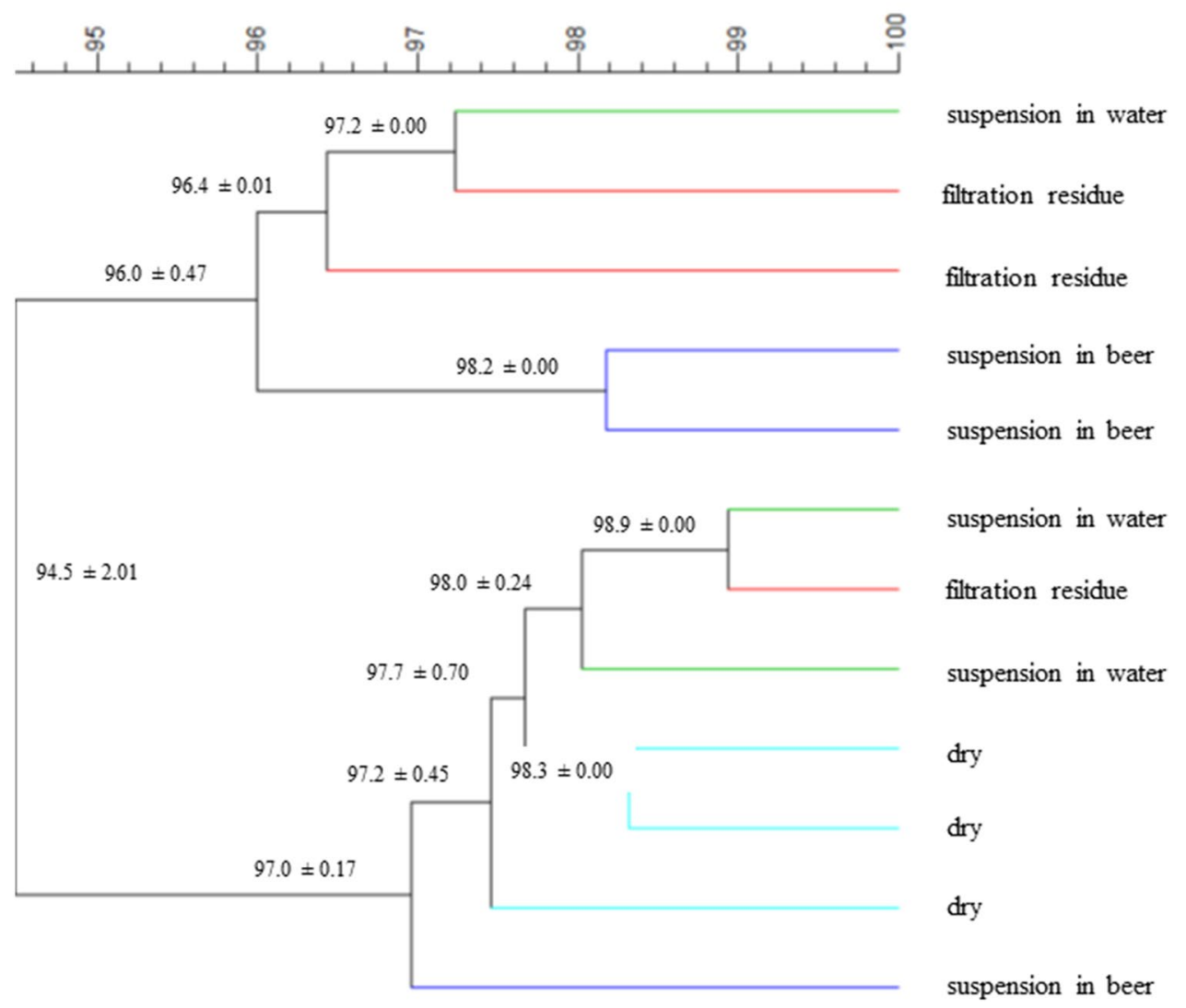


Fig. 6 Microscope images of silica gel (a), kieselguhr (b) and perlite particles (c) in bright field with white light source at 60x magnification and Raman spectra of silica gel (1), perlite (2), kieselguhr (3) and borosilicate glass (4) (colour-coded), $n=10, \lambda=532 \mathrm{~nm}$
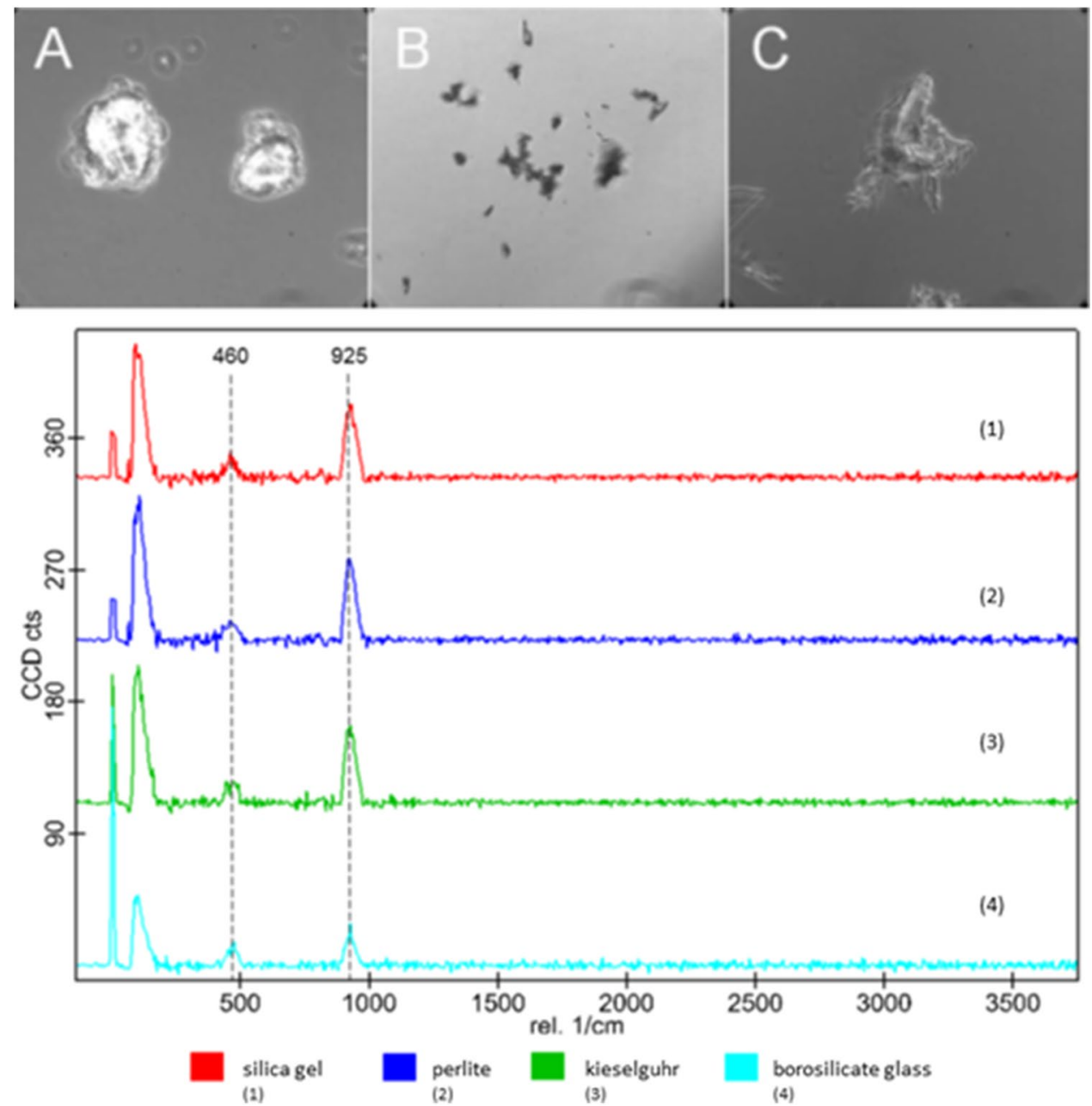

\section{PVPP (polyvinylpolypyrrolidone)}

Table 4 Silicon dioxide contents of various materials in $\%$

\begin{tabular}{ll}
\hline & $\mathrm{SiO}_{2}$ content [\%] \\
\hline Hydrated xerogel (Stabifix 2015) & $55-62$ \\
Flux-calcined kieselguhr (Römpp 2007) & $88-92$ \\
Perlite (Römpp 2010) & $68-75$ \\
Borosilicate (Römpp 2009a) & $70-80$ \\
\hline
\end{tabular}

any tendency to cluster formation. This is illustrated by the hierarchical cluster analysis, as shown in Fig. 7.

Due to the similarity of the silica gel, kieselguhr and perlite spectra to those of borosilicate glass, it is not possible to judge whether the spectra reflect the material properties of the particles or the slide. For this reason, no further analyses of these particles in different media were carried out. For future measurements, slides made of other materials such as calcium fluoride $\left(\mathrm{CaF}_{2}\right)$ should be used instead of borosilicate [20,21].
Figure 8 presents a microscope image of PVPP particles at $60 \times$ magnification and the corresponding spectrum of PVPP at different sample preparations. The microscope images show particle agglomerates of different sizes. When viewed purely optically there are no differences between the two preparations. The spectra each show a prominent peak at approx. $2930 \mathrm{rel} . \mathrm{cm}^{-1}$, as well as a region with several consecutive peaks at approx. 700-1700 rel. $\mathrm{cm}^{-1}$. The peak at approx. $2930 \mathrm{rel} . \mathrm{cm}^{-1}$ can be assigned to the oscillations of the $\mathrm{C}-\mathrm{H}$ compounds (2800-3100 $\mathrm{rel} \mathrm{cm}^{-1}$ ) [19]. C-H compounds are found in PVPP both in the hydrocarbon chain as well as in the cyclic amide structure. The oscillations of the $\mathrm{N}-\mathrm{H}$ compounds, as well as the carbonyl group from the amide group of PVPP, show peaks in the Raman spectrum at a wave number of about 1550-1700 rel. cm ${ }^{-1}$ [17]. Especially the carbonyl group, which is usually located at approx. $1700 \mathrm{rel} . \mathrm{cm}^{-1}$, can be identified in the spectra shown at a wavelength of approx. 1660 rel. $\mathrm{cm}^{-1}[17,22]$. This peak is lower in the samples 
Fig. 7 Evaluation of Raman spectra of silica gel, perlite, kieselguhr and borosilicate glass using a hierarchical cluster analysis, $\lambda=532 \mathrm{~nm}$

Fig. 8 Microscope images of PVPP particles in bright field with white light source at $\times 100$ magnification and Raman spectra of PVPP at different sample preparations: filtration residue (1), suspension in beer (2), suspension in water (3), dry (4) (colour-coded)); $n=10$, $\lambda=532 \mathrm{~nm}$
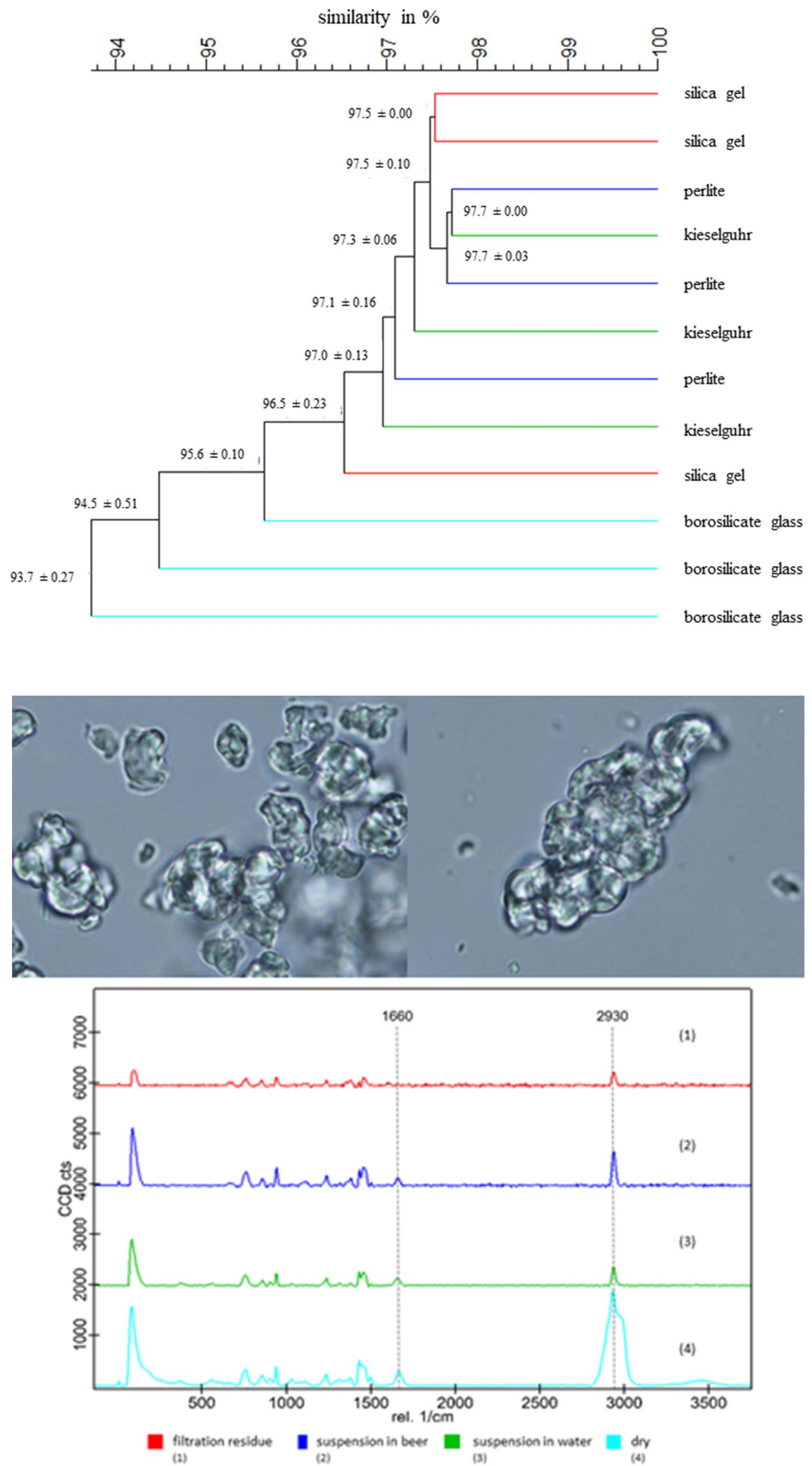
from the filtration residue compared to the other samples. This could be due to a shift in charge conditions at the carbonyl group caused by the hydrogen bonds that form there with the polyphenols. The remaining peaks in the range of approx. $700-1700$ rel. $\mathrm{cm}^{-1}$ were generally assigned in the literature to aliphatic hydrocarbons $\left(600-1300 \mathrm{rel} . \mathrm{cm}^{-1}\right)$ and organic carbon (1300-1700 rel. $\left.\mathrm{cm}^{-1}\right)[17,19]$. The bands below 1800 rel. $\mathrm{cm}^{-1}$ [19] and 1500 rel. $\mathrm{cm}^{-1}$ [17] also characterise the molecule as a whole. This range is also referred to as the "fingerprint" of the molecule [17, 19, 22].

Figure 8 also shows that the dry state spectrum differs from that in suspension (beer and water) and from the filtration residue. The peak at a wave number of approx. 2930 rel. $\mathrm{cm}^{-1}$ is particularly noticeable here, being higher and wider in the dry sample compared to the other samples. The Raman spectra of PVPP in dry state are at least $96.8 \%$ similar, whereas the similarities to the spectra of the other samples are between 51.2-59.9\%. Furthermore, the spectra of the other samples also show high similarities. The spectra of samples suspended in water are at least $99.6 \%$ similar, those of samples suspended in beer at least $95.2 \%$ similar and those from the filtration residue at least $89.4 \%$ similar. Furthermore, it can be seen that the samples from the filtration residue and those suspended in beer are at least $86.7 \%$ similar and that the samples suspended in water are at least $83.0 \%$ similar to those suspended in beer. However, when comparing the samples from the filtration residue with those from the water suspension, the spectra only show a similarity of $71.9-76.1 \%$. This may be attributed to polyphenol adsorption by the PVPP particles. Further analyses are necessary to verify this assumption. The similarity ratios of the Raman spectra can also be determined by means of a hierarchical cluster analysis: The cluster analysis shows four clusters, each consisting of the different particle variants. As in the similarity matrix, the cluster analysis shows only a low average similarity of $56.4 \%$ for the dry particles compared to the remaining particles, whereas the clusters of the remaining PVPP particles are, on average, at least $80.7 \%$ similar.

\section{Polymers}

Five individual spectra of each polymer type were recorded at several points within a sample and an average spectrum was generated. Reference spectra for the following experiments were to be recorded and spectral series were used to determine the intensity maxima of the polymers to empirically determine and confirm the general test parameters. For a better overview, only three of the respective individual spectra of the polymers are shown in Fig. 9.

The polymer samples 1-7 and 12 were the most common types of plastic and two additional polymer types (UP and PF). Finally, it was possible to generate the spectral data of

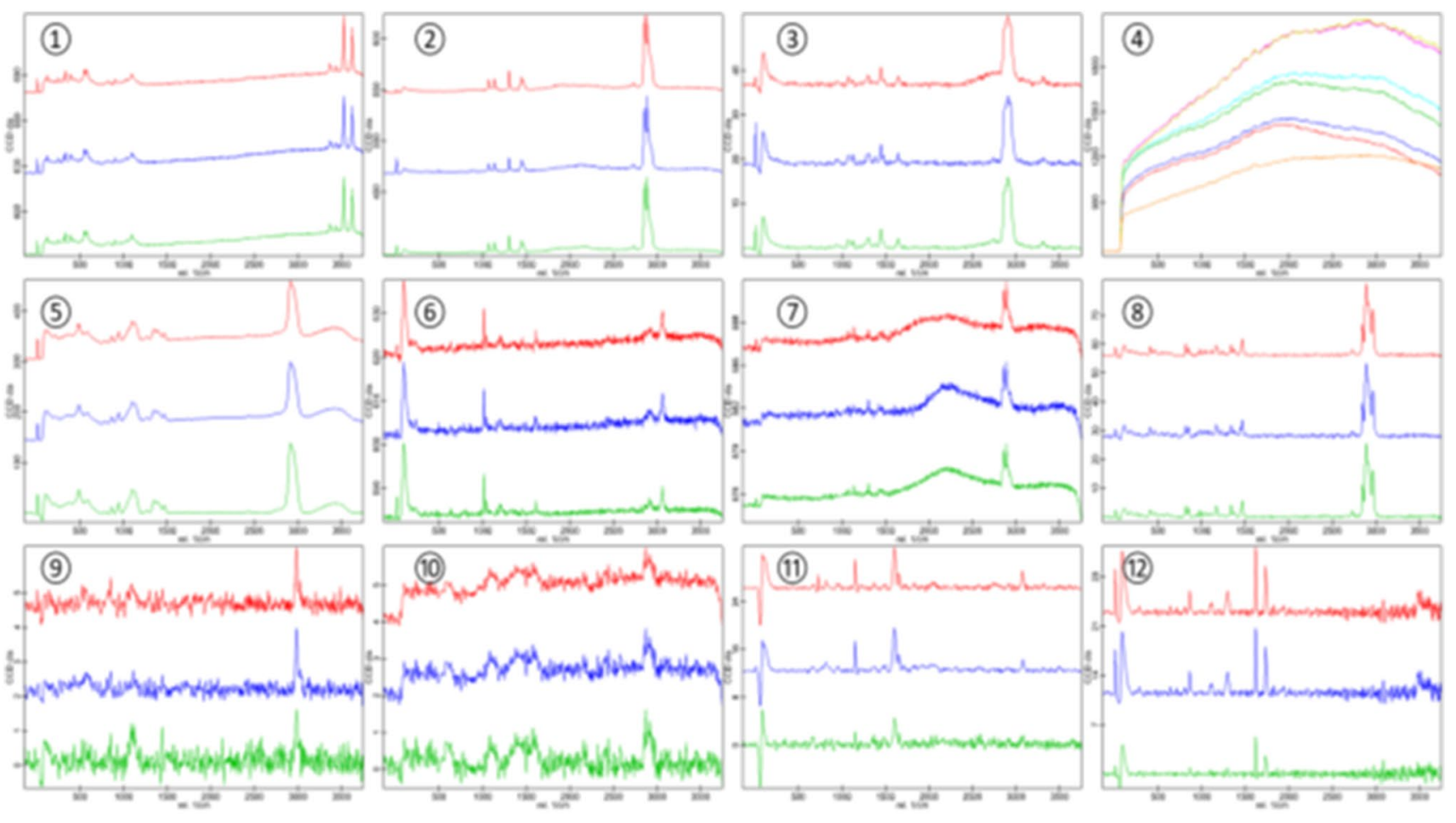

Fig. 9 Reference spectra of the polymer samples (1)=UP (2)=PA (3)=PE (4)=PF (5)=PMMA (6)=PS (7)=PVC (8)=PP (9)=PVDF (10)=PTFE (11) $=$ PEEK (12) $=$ PET) 
all polymers except the PF sample (4). However, due to fluorescence signals, the defined experimental parameters often had to be varied and adjusted accordingly. To get an overview of the laser intensity level at which the individual polymers tended to emit fluorescence signals, the intensity spectral series of the polymers were recorded. Figure 10 shows such an iterative spectral series. Using the standard parameters, the spectrum of the examined PA sample showed strong interference signals between 1500 and $4000 \mathrm{rel} . \mathrm{cm}^{-1}$. The laser intensity was then reduced and the number of accumulation cycles increased. The green graph shows, at $1 \mathrm{~mW}$ and 100 accumulation cycles, a maximum of peaks in the relevant fingerprint area at relatively low fluorescence signals. In the next iteration step $(0.5 \mathrm{~mW})$ the signal strength of the individual peaks decreased again, therefore the intensity optimum of this sample was set at $1 \mathrm{~mW}$.

With the help of the Sub BG function of the Project FIVE 5.1 software some of the fluorescence superimposed spectra could also be restored or cleaned up. However, the identifiability of the respective substance spectra also decreased. By means of this processing function, all acquired Raman signals are attenuated down to a common baseline. The substance-specific peaks should therefore be clearly identifiable in advance. Otherwise, the signal-to-noise ratio (SNR) may be too low, resulting in unidentifiable spectra. The partial images (9, (10) and (12) in Fig. 10 show polymer spectra which demonstrate a very low SNR value after using the Sub BG function. In the PF sample, the interference signals could not be reduced even at the lowest intensity level of $0.1 \mathrm{~mW}$ and ultimately led to the fact that the reference spectra could not be recorded. On the one hand, this may be due to the deep black colour of the sample, on the other hand, it may also be due to the unknown composition or possible additives. Specific information about the composition of the sample was not available, so this hypothesis remains unconfirmed. The microscopic examination showed no thermal damage whatsoever to the particles examined. Other samples were also dark or black in colour (e.g. PVC, PEEK) and yet the Raman spectra of these samples could be detected, even though they were very weak. It must therefore be assumed that unknown fluorophoric substances are present in the PF sample. In the end, the PF sample was the only polymer sample from which no spectra could be recorded and was therefore not investigated further in the subsequent experiments. Based on the results of the filter validations, the range of laser intensity levels to be tested was set at 0.1 to $10 \mathrm{~mW}$. The recorded spectra had to be clear enough to be used to identify the polymers and at the same time not show any thermally induced impairment of the analysis results. The minimum value was set at the value at which the particle under investigation could be identified perfectly using the resulting spectrum.

\section{Conclusion}

In this study a method for the identification of particles in beverages was established, tested and validated using standard suspensions. The confocal Raman micro-spectroscopy (RMS) system served as the analytical method. To make the results reproducible and comparable, the standard test parameters were determined and tested empirically. The conception of the method included the preparation of particle standards, the design of a sampling
Fig. 10 Spectral series of the PA sample after iterative modification of the test parameters

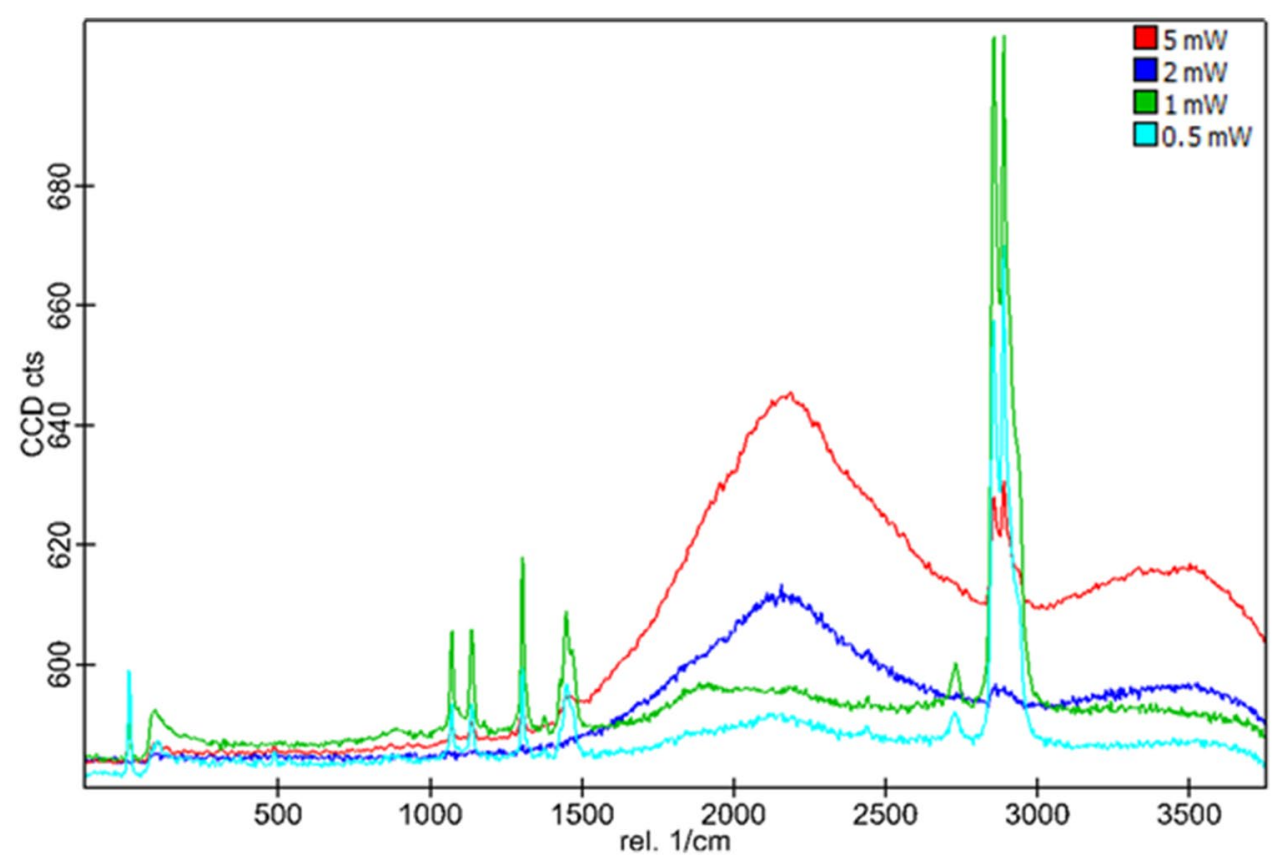


or preparation procedure, the selection of suitable membrane filters and the validation of the measuring method. Different filter aids and stabilising agents as well as different polymer samples of the most common types of plastics and some high-performance plastics from food production were investigated. The polymers were manually comminuted to generate the spectral data of the respective substances in the dry state. These served as reference spectra in later experiments. The substance-specific peaks were compared with corresponding literature values and included in the existing database. In addition, the thermal load capacity of each polymer sample was checked by iterative intensity increase of the laser power. Based on the given experimental parameters, a filtration apparatus was designed and manufactured. Different filter types were analysed to select suitable membrane filters. These differed in the carrier material, the filter thickness and the coating of the filter surface. The sampled filters were validated on the basis of a requirement profile created from the previous literature research. The filters were tested for their tendency to emit fluorescent signals and their thermal load capacity. This also served to empirically determine the analysis parameters necessary for the automatic analysis and identification of unknown particles. During the analysis of the particle samples on the selected membrane filters, small particles within the sample were manually located and focused. Video images and image scans of these particles were created, which were then evaluated using the programme functions Cluster Analysis and True Component Analysis. During the evaluation of the data, it was noticed that mainly polymer samples that tended to emit fluorescent signals could be detected by the Cluster Analysis. These primarily included dyed and pigmented polymers that exhibited strong fluorescence signals even at low intensity. The other polymers showed higher intensities only in some subranges or at significantly stronger laser settings, which were suitable for generating average spectra. To test the suitability of the analysis and the final selection of the most suitable membrane filter type, the spectra of the polymer particles on the previously validated filters were compared with the reference spectra of the dry polymer samples and presented in similarity matrices. In conclusion, the ability to characterise foreign particles, which can cause turbidity in beverages, using RMS has been successfully established.

Acknowledgements The authors want to thank Ludwig Gerlinger and Luis Raihofer for their excellent practical and theoretical work as part of their master's theses.

\section{Compliance with ethical standards}

Conflict of interest Eva-Maria Kahle, Martin Zarnkow and Fritz Jacob declare that they have no conflict of interest.
Compliance with ethics requirements The authors Eva-Maria Kahle, Martin Zarnkow and Fritz Jacob hereby confirm that this manuscript is performed according to and follows the COPE guidelines and has not already been published nor is it under consideration for publication elsewhere. This article does not contain any studies with human or animal subjects.

Funding Open Access funding enabled and organized by Projekt DEAL.

Open Access This article is licensed under a Creative Commons Attribution 4.0 International License, which permits use, sharing, adaptation, distribution and reproduction in any medium or format, as long as you give appropriate credit to the original author(s) and the source, provide a link to the Creative Commons licence, and indicate if changes were made. The images or other third party material in this article are included in the article's Creative Commons licence, unless indicated otherwise in a credit line to the material. If material is not included in the article's Creative Commons licence and your intended use is not permitted by statutory regulation or exceeds the permitted use, you will need to obtain permission directly from the copyright holder. To view a copy of this licence, visit http://creativecommons.org/licenses/by/4.0/.

\section{References}

1. Basarova G (1990) The structure-function relationship of polymeric sorbents for colloid stabilization of beer. Food Struct 9(3):1

2. Hartmann K, Kreisz S, Zarnkow M et al (2004) Identifizierung von Filterhilfsmitteln nach den einzelnen Filtrationsschritten. Der Weihenstephaner 4:141-143

3. Leiper KA, Miedl M (2008) Colloidal stability of beer. Academic Press, Burlington

4. Steiner E, Becker T, Gastl M (2010) Turbidity and haze formation in beer-insights and overview. J Inst Brew 116(4):360-368. https ://doi.org/10.1002/j.2050-0416.2010.tb00787.x

5. Sharpe FR, Channon PJ Beer Haze Caused by Can Lid Lubricant. Institute of Brewing 1987(Vol. 93): 163

6. Oßmann BE, Sarau G, Holtmannspötter H et al (2018) Small-sized microplastics and pigmented particles in bottled mineral water. Water Res 141:307-316

7. Kaiser W (2015) Kunststoffchemie für Ingenieure: Von der Synthese bis zur Anwendung. Carl Hanser Verlag GmbH Co KG

8. Schymanski D, Goldbeck C, Humpf H-U et al (2018) Analysis of microplastics in water by micro-Raman spectroscopy: release of plastic particles from different packaging into mineral water. Water Res 129:154-162. https://doi.org/10.1016/j.watre s.2017.11.011

9. Arthur C, Baker J, Bamford H (2008) International research workshop on the occurrence, effects, and fate of microplastic marine debris. In: Conference Proceedings, pp 9-11

10. Mastanjević K, Krstanović V, Lukinac J et al (2018) Beer-the importance of colloidal stability (non-biological haze). Fermentation 4(4):91

11. Steiner E, Gastl M, Becker T (2011) Protein changes during malting and brewing with focus on haze and foam formation: a review. Eur Food Res Technol 232(2):191-204. https://doi.org/10.1007/ s00217-010-1412-6

12. Steiner E, Gastl M, Becker T (2011) Die Identifizierung von Trübungen in Bier (1). Brauwelt 2011(Ausgabe 05/06): 161-166

13. Steiner E, Gastl M, Becker T Die Identifizierung von Trübungen in Bier (2). Brauwelt 2011(Ausgabe 7): 193-205

14. Steiner E, Arendt EK, Gastl M et al (2011) Influence of the malting parameters on the haze formation of beer after filtration. Eur 
Food Res Technol 233(4):587-597. https://doi.org/10.1007/s0021 7-011-1547-0

15. Kahle E-M, Zarnkow M, Jacob F (2019) Substances in beer that cause fluorescence: evaluating the qualitative and quantitative determination of these ingredients. Eur Food Res Technol 7(06): 193. https://doi.org/10.1007/s00217-019-03394-x

16. Erni-Cassola G, Gibson MI, Thompson RC et al (2017) Lost, but found with Nile Red: a novel method for detecting and quantifying small microplastics ( $1 \mathrm{~mm}$ to $20 \mathrm{\backslash mum}$ ) in environmental samples. Environ Sci Technol 51(23):13641-13648

17. Smith E, Dent G (2005) Modern Raman spectroscopy: a practical approach. Hoboken, John Wiley \& Sons

18. Anger PM, von der Esch E, Baumann T et al (2018) Raman microspectroscopy as a tool for microplastic particle analysis. TrAC, Trends Anal Chem 109:214-226
19. Dieing T, Hollrichter O, Toporski J (2011) Confocal raman microscopy. Springer, Berlin, Heidelberg

20. El-Mashtoly SF, Petersen D, Yosef HK et al (2014) Label-free imaging of drug distribution and metabolism in colon cancer cells by Raman microscopy. Analyst 139(5):1155-1161

21. Tolstik T, Marquardt C, Matthäus C et al (2014) Discrimination and classification of liver cancer cells and proliferation states by Raman spectroscopic imaging. Analyst 139(22):6036-6043

22. Smith E, Dent G (2013) Modern Raman spectroscopy: A practical approach. John Wiley \& Sons

Publisher's Note Springer Nature remains neutral with regard to jurisdictional claims in published maps and institutional affiliations. 